\title{
APROXIMACIÓN A LA REALIDAD DE LOS DESAHUCIOS. PERFIL Y CARACTERÍSTICAS DE LAS FAMILIAS EN PROCESO DE DESAHUCIOS EN LA CIUDAD DE MÁLAGA
}

\author{
An approach to the reality of evictions. Profile and characteristics of \\ families in the process of eviction in the city of Málaga
}

\author{
RAFAel ARredondo Quijada ${ }^{1}$ y María de las Olas Palma García ${ }^{2}$
}

\section{Resumen}

El incremento de familias que se encuentran en situación de vulnerabilidad y/o riesgo social ante la pérdida de su vivienda se presenta en la actualidad como realidad emergente para la intervención social. Con el objetivo de acercarnos a dicha realidad, el presente artículo recoge los resultados de una investigación desarrollada en el marco del Master de Investigación e Intervención Social y Comunitaria de la Universidad de Málaga, en la que se analizan los hechos ocurridos en los últimos años, como posibles explicaciones de los actuales desahucios y las características de las familias que los sufren. En el estudio participan 110 sujetos, identificados de forma aleatoria a partir de las familias que acuden a los diferentes recursos de atención a la realidad de los desahucios en la ciudad de Málaga, principalmente a través de la Plataforma de Afectados por la Hipoteca (PAH). A los participantes se les aplica un cuestionario específico diseñado para esta investigación que explora el perfil de las familias afectadas por los desahucios y las circunstancias que presentan ante el impago de sus hipotecas. Los resultados muestran el desempleo como principal causa de dicho impago, a la vez que se describen como posibles consecuencias, efectos directos sobre el bienestar psicosocial de las familias implicadas. A partir de los resultados, se realizan propuestas para la intervención con dichas familias y su entorno, así como con las entidades financieras, tales como la necesidad de abordar el desempleo como circunstancia fundamental que está originando los impagos hipotecarios, la utilización de la mediación como instrumento de resolución del conflicto y la necesidad de que se realicen las modificaciones legislativas correspondientes a fin de que la vivienda se considere un derecho fundamental. Se concluye sobre la necesidad de seguir con esta línea de investigación ante la escasez de estudios que aborden esta problemática

Palabras clave: Desahucio, hipoteca, vivienda, vulnerabilidad, Plataforma de Afectados por la Hipoteca (PAH).

\section{Abstract}

The increase in families who are vulnerable and / or at social risk at the loss of their home is presented today as emerging reality for social intervention. In order to approach this reality, this article presents the results of a research project developed within the framework of the Master of Research and Social Intervention and Community at the University of Málaga, which discusses the events of recent years, as possible explanations for the current evictions and the characteristics of families who suffer. The study involved 110 subjects, randomly identified from the families that use the different resources for attention about the reality of eviction in the city of Málaga, primarily through the Platform of People Affected by Mortgages (PAH). Participants are given a specific questionnaire designed for this research that explores the profile of the families affected by the evictions and the circumstances that present themselves defaulting on their mortgages. The results show unemployment as the main cause of such failure, while described as potential consequences, direct effects on psychosocial well-being of the families involved. From the results, proposals are made for intervention with these families and their environment, as well as financial institutions, such as the need to tackle unemployment as fundamental circumstance that is causing mortgage defaults, the use of mediation as instrument of conflict resolution and the need for legislative changes to be made so that appropriate housing is considered a basic right. We conclude on the need to continue this line of research due to the scarcity of studies addressing this problem.

Keywords: Eviction, mortgage, housing, vulnerability, Platform of People Affected by Mortgages (PAH).

1. Asociación Cívica para la Prevención (ACP), Málaga, España. Responsable de la correspondencia. C/ Alfeizar núm. 1. 29130 Alhaurín de la Torre. Málaga, España. E-mail: rafael.arredondo. quijada@cgtrabajosocial.es

2. Universidad de Málaga. Departamento de Psicología Social, Antropología Social, Trabajo Social y Servicios Sociales. Facultad de Estudios Sociales y del Trabajo. Málaga, España 


\section{Introducción}

Hablar en la actualidad del problema de vivienda nos lleva de lleno a tratar la situación de las personas que ante la imposibilidad de hacer frente a su hipoteca, se ven inmersas en un proceso de cambio en el que tienen que afrontar nuevas situaciones y roles, que en muchos de los casos, termina con el desahucio de su vivienda. Es este un contexto emergente para la intervención social, en el que de acuerdo con Navarro (2005) se ha de considerar «la privación de una vivienda digna como una de las manifestaciones más visibles de la pobreza y la exclusión social» (p.175). De ahí que cuando se interviene en procesos de exclusión, la vivienda es un elemento que junto al empleo, la formación o el acompañamiento social, se consideran fundamentales. (Concurso de Buenas Prácticas. Dubai 1996).

Ante este nuevo escenario, actualmente constituido en uno de los dramas más difíciles y preocupantes para miles de familias en el conjunto de nuestra sociedad, es necesario desarrollar «estudios que proporcionen criterios para la construcción de índices que permitan no sólo conocer la extensión de la pobreza y la exclusión, sino también sus factores determinantes» (Navarro, 2005, p.175).

La revisión bibliográfica realizada en el marco de esta investigación ha puesto de manifiesto la falta de estudios específicos sobre esta problemática, como posible consecuencia de que hasta ahora esta realidad no se había visibilizado suficientemente, ni había afectado de una manera tan contundente como está ocurriendo en los últimos años, sobre todo desde el 2007 a raíz de la crisis financiera y del estallido de la llamada burbuja inmobiliaria.

No es hasta noviembre de 2012, cinco años después del inicio de la crisis, cuando el Centro de Investigaciones Sociológicas (CIS) incorpora en sus encuestas el concepto desahucios, a la hora de preguntar por los tres principales problemas en España. En base a ello, según el barómetro de abril de 2013, los desahucios se convierten en el sexto problema más importante indicado por los españoles, por delante incluso de problemas como la educación, los recortes, la inmigración, las pensiones, el fraude fiscal o el terrorismo.

Da la sensación de que los desalojos que se producen a cientos de familias, ha cogido al conjunto de la sociedad, y sobre todo a los sistemas de protección, desconcertados y sin capacidad para encontrar soluciones que den respuestas concretas e inmediatas a una emergencia como tal. Dichos desalojos conllevan no sólo «la vergüenza, el estigma y el miedo asociado al proceso» (Colau y Alemany, 2012, p. 16), sino todo un conjunto de problemas colaterales, que quedan solapados por la inmediatez de dar respuesta a la búsqueda de una nueva vivienda o lugar de alojamiento transitorio. 
Esos problemas colaterales son en sí mismos situaciones multiproblemáticas que se encuentran en la base de la intervención social. Elementos como el absentismo escolar, en caso de que haya menores, efectos sobre la salud física y psíquica, conflictos en las relaciones familiares, falta de empleo o precarización en la contratación, etc., «son agravantes en cuanto a costes sociales que incidirán en la vulnerabilidad para iniciar un proceso de exclusión social» (Laparra, et al., 2007, p. 45).

Todo este escenario de vulnerabilidad social, según el reciente Catálogo de Referencia de Servicios Sociales ${ }^{3}$, sigue siendo objeto de análisis e intervención para los profesionales del Trabajo Social como profesionales de referencia, lo que plantea nuevos retos para la disciplina ante la realidad de los desahucios. Es necesario reflexionar sobre si hubiera sido posible haber evitado, o en todo caso, haber pronosticado las consecuencias que ahora están manifestándose. A ello quiere contribuir el presente artículo, aportando un primer acercamiento ante la realidad de los desahucios que ofrezca alternativas de intervención, y líneas futuras de investigación.

\section{La realidad de los desahucios: pasado y presente}

\section{Perfil del mercado inmobiliario en España}

El desenlace de esta situación, que afecta a familias con nombres y apellidos, tiene su origen en circunstancias que se han venido produciendo históricamente, tales como el incremento y fomento de la compra sobre el alquiler de manera espectacular por parte de los ciudadanos españoles.

El Plan Estatal de fomento del alquiler de vivienda, la rehabilitación edificatoria, y la regeneración y renovación urbanas, 2013-2016, establece que el alquiler significa en España el 17\% frente al 83\% del mercado de la vivienda en propiedad, mientras que en Europa, en porcentajes medios, el mercado de la vivienda principal en alquiler representa el 38\%, frente al 62\% de vivienda en propiedad.

Estos datos reflejan una tendencia a la propiedad en las últimas décadas, que no siempre ha estado presente en nuestro país. De hecho, en 1950 el 51\% de la población vivía de alquiler y la cifra superaba el $90 \%$ en grandes ciudades como Madrid o Barcelona (Colau y Alemany, 2012). Aun así, parafraseando al primer ministro de la vivienda en 1957 José Luis Arrese, esto no respondía a los intereses de gobierno, ya que se quería «un país de propietarios no de proletarios, dado que el hombre, cuando no tiene hogar, se apodera de la

3. BOE 117 de 16 de mayo 2013 
calle, y perseguido por su mal humor, se vuelve subversivo, agrio, violento...» (Citado por Colau y Alemany, 2012 p. 34).

En paralelo se ha producido un incremento espectacular en la construcción de vivienda de promoción libre frente a la vivienda de protección. Según el Instituto Nacional de Estadísticas (INE) ${ }^{4}$, en el período 1997 a 2012, se iniciaron 6.977 .176 viviendas, de las que el $85,6 \%$ son de protección y el 14,2\% son de promoción libre. Tal como se observa en el cuadro 1, desglosado este periodo con referencia al comienzo de la crisis financiera, se produce un descenso en la construcción de viviendas, finalizando con ello el llamado boom inmobiliario.

Cuadro I. Viviendas iniciadas según tipo, período 1997 - 2012

\begin{tabular}{|l|c|c|c|c|}
\hline \multirow{2}{*}{ Viviendas iniciadas } & \multicolumn{2}{|c|}{ Período 1997-2006 } & \multicolumn{2}{c|}{ Período 2007-2012 } \\
\cline { 2 - 5 } & & $\%$ sobre total & & $\%$ sobre total \\
\hline Protección & 631.796 & $11,23 \%$ & 365.748 & $27,08 \%$ \\
\hline Promoción libre & 4.994 .516 & $88,77 \%$ & 985.116 & $72,92 \%$ \\
\hline Total & 5.626 .312 & $100,00 \%$ & 1.350 .864 & $100,00 \%$ \\
\hline
\end{tabular}

Elaboración propia. Fuente: Instituto Nacional de Estadística

Según el Censo de población y viviendas de 2011 del INE ${ }^{5}$, España dispone de 25.208 .623 viviendas, de ellas el $13,66 \%$ se encuentran vacías.

\section{Perfil del mercado hipotecario}

Como consecuencia de este incremento de construcción de viviendas de promoción libre, las entidades financieras (Cajas de Ahorros y Bancos) han hecho de los productos hipotecarios su principal negocio. De acuerdo con Trabada (2012, p.177), «que los créditos hipotecarios pasasen a ser el negocio principal para las entidades financieras derivó en un abandono de $<<$ buenas prácticas hipotecarias $>>$, como que la cuota a pagar no se elevase por encima del umbral de un tercio de los ingresos del hogar endeudado, y que la cantidad económica del préstamo no superase el $80 \%$ del valor de tasación de la vivienda hipotecada.»

4. http://www.ine.es/jaxi/tabla.do? path=/t38/bme2/t07/a081/10/\&file=1300007. px\&type $=$ pcaxis $\& \mathrm{~L}=0$

5. http://www.ine.es/prensa/np775.pdf 
En este escenario, según los datos del Registro de la Propiedad ${ }^{6}$, en los últimos diez años se ha producido en España una media anual de más de 870.000 operaciones de hipotecas constituidas. Según la Encuesta de Condiciones de $\mathrm{Vida}^{7}$ correspondiente al año 2012, el 29,9\% de los hogares posee vivienda en propiedad pero con una hipoteca pendiente. Ante este dato, el Banco de España ${ }^{8}$ indica en su informe del 10 de mayo de 2013, que a fecha del 31 de diciembre de 2012 de las viviendas hipotecadas, el 90,7\% correspondía a la vivienda habitual, y sólo un 9,2\%, a hipotecas sobre otras viviendas.

No sólo las entidades financieras generaron ante las hipotecas un negocio sin reservas y precauciones, sino que también los ayuntamientos vieron una gran oportunidad de obtención de ingresos financieros a través del conjunto de la actividad relacionada con el mercado inmobiliario: licencias y convenios urbanísticos, tasas e impuestos sobre construcciones, instalaciones y obras, enajenación de suelo público, etc. Tomando como ejemplo el Ayuntamiento

Cuadro II. Ingresos relacionados al ámbito inmobiliario según presupuesto Ayuntamiento de Málaga 2006 y 2013

\begin{tabular}{|l|r|r|}
\hline Concepto & $\begin{array}{c}\text { Presupuesto año 2006 } \\
\text { (euros) }\end{array}$ & $\begin{array}{c}\text { Presupuesto año 2013 } \\
\text { (euros) }\end{array}$ \\
\hline Licencias urbanísticas & $14.000 .000,00$ & $5.642 .473,92$ \\
\hline Convenios urbanísticos & $93.722 .059,00$ & 0,00 \\
\hline Construcciones, instalaciones y obras & $20.203 .300,00$ & $4.478 .230,00$ \\
\hline Enajenación de parcelas vía pública & $28.148 .642,00$ & $30.000,00$ \\
\hline Enajenación de otros terrenos & $75.000,00$ & $1.525 .000,00$ \\
\hline $\begin{array}{l}\text { Enajenación de viviendas y locales de } \\
\text { negocio }\end{array}$ & $16.792 .309,78$ & $1.789 .337,11$ \\
\hline Enajenación de otras inversiones reales & $38.238 .769,42$ & $1.438 .563,12$ \\
\hline Total ingresos ámbito inmobiliario & $211.180 .080,20$ & $\mathbf{1 4 . 9 0 3 . 6 0 4 , 1 5}$ \\
\hline Ingresos totales previstos & $958.036 .278,52$ & $685.132 .295,78$ \\
\hline $\begin{array}{l}\text { \% Ingresos ámbito inmobiliario sobre el } \\
\text { total }\end{array}$ & $\mathbf{2 2 , 0 4 \%}$ & $\mathbf{2 , 1 8 \%}$ \\
\hline
\end{tabular}

Elaboración propia. Fuente: Presupuestos Ayuntamiento de Málaga

6. http://www.registradores.org/get?DS=6\&file=/Estadisticas/Panorama_registral_ de_impagos_hipotecarios_de_vivienda_en_2012.pdf

7. http://www.ine.es/prensa/np740.pdf

8. http://www.bde.es/f/webbde/GAP/Secciones/SalaPrensa/NotasInformativas/Briefing_notes/es/notabe10-05-13.pdf 
de Málaga ${ }^{9}$, según se observa en el cuadro 2, en el presupuesto para el año 2006 , previo a la crisis financiera, el 22,04\% de los ingresos previstos provenían de los conceptos anteriormente indicados, mientras que para el presupuesto del año 2013 en los mismos ítems, la previsión de ingreso sobre el total sólo representaba el 2,18\%, un 92,94 menos.

\section{Capacidad de endeudamiento de las familias y vulnerabilidad económica}

La privación de una vivienda puede situar a una familia en las puertas de la vulnerabilidad, y a un paso de la exclusión social (Navarro, 2005). Esta circunstancia ha sido el elemento utilizado, tanto por la administración central como por parte de los gobiernos autonómicos, a la hora de aprobar normativas para intervenir sobre las personas que se ven afectadas por un desahucio. En concreto, las últimas normativas aprobadas sobre medidas ante deudores hipotecarios, hacen explícitos los indicadores de riesgo que se tienen en cuenta para considerar a una familia en el umbral de la exclusión social, como colectivo especialmente vulnerable, o como persona en una situación de emergencia o exclusión social (ver cuadro 3).

Con esta delimitación de familias y otras muchas, que aun no encontrándose entre los parámetros aquí expuestos, también pueden presentar dificultades para hacer frente a la cuota hipotecaria, se establece el punto de partida para que la entidad financiera decida interponer una demanda vía judicial de reclamación de pagos pendiente, iniciándose con ello una ejecución hipotecaria. Este proceso dará pie a una posterior subasta de la vivienda si no se han satisfecho las deudas reclamadas, y al denominado lanzamiento, acto por el que la familia es sacada a la fuerza, en caso necesario, de la que ha sido su vivienda.

En paralelo, como consecuencia del estallido de la burbuja inmobiliaria a raíz de la crisis financiera, se están produciendo cifras de paro jamás registradas. En el primer trimestre de 2007, inicio de la crisis, se situaba en 413.300 los hogares del conjunto del estado que tenían todos sus miembros en paro. Seis años después, en el primer trimestre de 2013 alcanza la cifra de 1.906 .100 hogares, lo que supone un incremento del 461,19\%, según la Encuesta de Población Activa (EPA) ${ }^{10}$.

9. http://presupuestos2013.malaga.eu/export/sites/default/economia/presupuestos2013/ portal/menu/seccion_0001/documentos/estado_consolidado.pdf

10. http://www.ine.es/jaxi/menu.do? L=0\&type=pcaxis\&path=\% $2 \mathrm{Ft} 22 \% 2$ Fe308_mnu\&file=inebase 
Cuadro III. Perfil de deudores hipotecarios en riesgo según normativas

\begin{tabular}{|c|c|}
\hline Normativa & Requisitos beneficiarios \\
\hline $\begin{array}{l}\text { Real Decreto-ley } 6 / 2012 \text {, de } 9 \\
\text { de marzo, de medidas urgentes } \\
\text { de protección de deudores } \\
\text { hipotecarios sin recursos } \\
\text { (Gobierno central) } \\
\text { Referido a deudores en el } \\
\text { umbral de exclusión }\end{array}$ & $\begin{array}{l}\text { a. Vivienda habitual } \\
\text { b. Inexistencia de otra vivienda } \\
\text { c. Miembros de la unidad familiar sin rentas } \\
\text { d. Cuota hipotecaria superior al } 60 \% \text { de los ingresos } \\
\text { netos del conjunto de la unidad familiar } \\
\text { e. Miembros de la unidad familiar sin otros bienes } \\
\text { o derechos patrimoniales suficientes con los que } \\
\text { hacer frente a la deuda }\end{array}$ \\
\hline $\begin{array}{l}\text { Ley } 1 / 2013, \text { de } 14 \text { de mayo, } \\
\text { de medidas para reforzar la } \\
\text { protección a los deudores } \\
\text { hipotecarios, reestructuración } \\
\text { de deuda y alquiler social } \\
\text { (Gobierno central) } \\
\text { Real Decreto-ley } 27 / 2012 \text {, de } \\
15 \text { de noviembre, de medidas } \\
\text { urgentes para reforzar la } \\
\text { protección a los deudores } \\
\text { hipotecarios } \\
\text { (Gobierno central) } \\
\text { Referidos a colectivos } \\
\text { especialmente vulnerables }\end{array}$ & $\begin{array}{l}\text { a. Familia numerosa } \\
\text { b. Unidad familiar monoparental con dos hijos a cargo } \\
\text { c. Unidad familiar con un menor de tres años } \\
\text { d. Unidad familiar con algún miembro con } \\
\text { discapacidad (mayor 33\%), dependencia o } \\
\text { enfermedad. O que conviva en la misma vivienda } \\
\text { con el titular de la hipoteca o su cónyuge por } \\
\text { vínculo de parentesco hasta el tercer grado de } \\
\text { consaguinidad o afinidad } \\
\text { e. Deudor hipotecario desempleado y sin prestaciones } \\
\text { f. Unidad familiar con víctima de violencia de género } \\
\text { g. Ingresos de la unidad familiar no superen } 3 \text { veces } \\
\text { el IPREM } \\
\text { h. En los cuatro años anteriores a la solicitud la } \\
\text { unidad familiar haya sufrido una alteración } \\
\text { significativa de sus circunstancias económicas en } \\
\text { términos de esfuerzo de acceso a la vivienda } \\
\text { i. Cuota hipotecaria superior al } 50 \% \text { de los ingresos } \\
\text { netos del conjunto de la unidad familiar } \\
\text { j. Disponer de una sola vivienda } \\
\text { k. La carga hipotecaria sobre la renta familiar se haya } \\
\text { multiplicado por al menos } 1,5\end{array}$ \\
\hline $\begin{array}{l}\text { Decreto-ley } 6 / 2013 \text {, de } 9 \text { de } \\
\text { abril, de medidas para asegurar } \\
\text { el cumplimiento de la Función } \\
\text { Social de la Vivienda (Junta de } \\
\text { Andalucía) } \\
\text { Referido a personas en situación } \\
\text { de emergencia o exclusión social }\end{array}$ & $\begin{array}{l}\text { a. La carga hipotecaria sobre la renta familiar se haya } \\
\text { multiplicado por, al menos, 1,5 y suponga más de } \\
\text { un tercio de los ingresos familiares. } \\
\text { b. Ingresos de la unidad familiar no supere } 3 \text { veces el } \\
\text { IPREM } \\
\text { c. Informe de los Servicios Sociales Comunitarios } \\
\text { sobre la situación de emergencia o exclusión social } \\
\text { en que puede quedar el beneficiario en caso de } \\
\text { lanzamiento }\end{array}$ \\
\hline
\end{tabular}

Elaboración propia. Fuente: Boletín Oficial del Estado y Boletín Oficial de la Junta de Andalucía 
No podemos olvidar que la tasa de riesgo de pobreza según las diferentes Encuestas de Condiciones de Vida del INE ${ }^{11}$, recogidas desde 2004 hasta 2012, ha experimentado un crecimiento del 1,2\%, pasando del 19,9\% en el año 2004 a un $21,1 \%$ en el año 2012, generándose una cada vez mayor bolsa de familias con dificultades para hacer frente de manera puntual, a los compromisos contraídos con las entidades financieras.

Por último, y según los datos facilitados por Cáritas en su VIII Informe del Observatorio de la Realidad Social (2013), «las principales causas de empeoramiento de la situación económica son, por este orden, el desempleo y la precarización de las condiciones laborales; y el endeudamiento de los hogares (especialmente hipotecas de vivienda)». Ante ello, la ayuda más habitual de apoyo desde las familias, está relacionada con ayudas económicas para la vivienda.

\section{Proceso de ejecución hipotecaria}

Todas estas circunstancias empiezan a estar visibilizadas a nivel estadístico, principalmente por el informe que cada tres meses elabora el Consejo General del Poder Judicial, titulado «Datos sobre el efecto de la crisis en los órganos judiciales», en el que dedica una serie de apartados a las ejecuciones hipotecarias y lanzamientos ${ }^{12}$. En él se refleja cómo se ha producido desde el año 2008 hasta finales de 2012, un total de 415.117 ejecuciones hipotecarias, y 236.973 lanzamientos en el conjunto del Estado. A su vez, el propio Consejo General del Poder Judicial en su memoria anual «La justicia dato a dato», viene recogiendo desde el año 2008, el número de lanzamientos sobre los recibidos que son realizados de manera positiva ${ }^{13}$ (ver cuadro 4 ).

Tal como refleja el cuadro 4, se mantiene un incremento en cuanto a los lanzamientos cumplimentados positivamente año tras año, en un 166,21\% más del año 2008 al 2012. Aunque no existen datos sobre qué circusntancias

11. http://www.ine.es/jaxi/tabla.do?path=/t25/p453/provi/lo/\&file=01002. px\&type $=$ pcaxis $\& L=0$

12. Ejecuciones hipotecarias: Procedimientos tramitados en los Juzgados de Primera Instancia, que permiten exigir el pago de las deudas garantizadas por prenda o hipoteca al acreedor con escritura de hipoteca a su favor.

Lanzamientos: Diligencias practicadas por la comisión judicial que habitualmente implica el desplazamiento de funcionarios a las viviendas para el desalojo. Sólo se contabilizan los realizados por los servicios comunes de notificaciones y embargos y no los realizados directamente por los juzgados en las localidades en las que no existen dichos servicios.

13. Lanzamientos con cumplimiento positivo: Lanzamientos en los que se ha podido practicar el lanzamiento acordado por el juzgado. 
Cuadro IV. Ejecuciones hipotecarias, lanzamientos recibidos y lanzamientos cumplimentados positivamente en el Estado 2008-2012

\begin{tabular}{|c|c|c|c|c|}
\hline Año & $\begin{array}{c}\text { Ejecuciones } \\
\text { hipotecarias }\end{array}$ & $\begin{array}{c}\text { Lanzamientos } \\
\text { recibidos }\end{array}$ & $\begin{array}{c}\text { Lanzamientos } \\
\text { cumplimentados } \\
\text { positivamente }\end{array}$ & $\begin{array}{c}\text { \% lanzamientos } \\
\text { cumplimentados } \\
\text { positivamente sobre los } \\
\text { recibidos }\end{array}$ \\
\hline 2008 & 58.686 & 26.748 & 17.433 & $65,17 \%$ \\
\hline 2009 & 93.319 & 33.918 & 22.493 & $66.32 \%$ \\
\hline 2010 & 93.636 & 47.809 & 32.689 & $68,37 \%$ \\
\hline 2011 & 77.854 & 58.241 & 40.740 & $69,95 \%$ \\
\hline 2012 & 91.622 & 70.257 & 46.408 & $66,05 \%$ \\
\hline
\end{tabular}

Elaboración propia. Fuente Consejo General del Poder Judicial

son las que han impedido la realización de estos lanzamientos en su totalidad, es conocido que cada vez se están produciendo más actos ciudadanos para llegar a su paralización o retraso.

Recientemente, en abril y en mayo de 2013, se han publicado dos nuevos documentos referentes a información estadística sobre esta problemática. El primero de ellos corresponde al Colegio de Registradores de la Propiedad, Bienes Muebles y Mercantiles de España, bajo el título «Panorama Registral impagos hipotecarios de vivienda 2012». Dicho informe indica que durante el año 2012 se han registrado 38.976 adjudicaciones por ejecución hipotecaria sobre vivienda, ${ }^{14}$ relativos a titulares personas físicas. Estas adjudicaciones suponen el final del proceso de ejecución hipotecaria, que se inicia con la certificación registral.

El segundo documento corresponde a la nota hecha pública por el Banco de España con fecha del 10 de mayo, en el que se indica que el número total de entrega de viviendas ocurrido en el año 2012 ha sido de 39.167, de ellas, 32.490 vivienda habitual. En el $83 \%$ de los casos de entrega judicial de viviendas habituales, la vivienda estaba vacía, por lo que en un $17 \%$ de ellos, en el momento de dicha entrega había una familia ocupándola.

Tanto el Relator Especial de Naciones Unidas, Sr. Miloon Kothari, con su informe «Promoción y protección de todos los derechos humanos, civiles,

14. Adjudicaciones como consecuencia de procedimiento de ejecución hipotecaria relativas exclusivamente a vivienda: Operaciones que inscriben el cambio de titularidad como consecuencia del impago de la hipoteca. Pueden llegar con demora al Registro de la Propiedad por el retraso de las entidades financieras para evitar los gastos inherentes en tanto no venden a un tercero la vivienda, aprovechando en ese momento para hacer ambas inscripciones, adjudicación y venta. 
políticos, económicos, sociales y culturales incluido el derecho al desarrollo» del año 2008, como el informe «El derecho a una vivienda adecuada», presentado en la Asamblea General de las Naciones Unidas en el año 2012, han planteado que la vivienda es un derecho recogido no sólo a nivel constitucional, como es el caso español, sino también en el derecho internacional incorporado en la Declaración Universal de los Derechos Humanos. Se ha indicado, además, que urge una protección de aquellos sectores más vulnerables para que puedan acceder a una vivienda digna y habitable, en condiciones económicas posibles y viables. Por ello, cualquier acto de violencia y/o presión que se realiza para desalojar a una familia de su vivienda, debe desaparecer e incluso ser penalizado. Se defiende con ello, un rechazo a modelos en donde la corrupción y la especulación urbanística sitúe a la vivienda no como un derecho humano, sino como un instrumento de intercambio comercial para obtener beneficios empresariales, sobre todo con aquellas familias que tienen la necesidad de acceder a lo que consideramos como vivienda habitual.

A su vez el dictamen emitido por la abogada general del Tribunal de Justicia de la UE, Juliane Kokott, el 8 de noviembre de 2012, a raiz de una demanda de Mohamed Aziz contra CatalunyaCaixa, donde se recoge la incompatibilidad que se ha estado realizando respecto a la Directiva 93/13, indicando que: «Un sistema de ejecución de títulos notariales sobre bienes hipotecados o pignorados en el que las posibilidades de oposición frente a la ejecución se encuentran restringidas es incompatible con la Directiva 93/13/CEE del Consejo, de 5 de abril de 1993, sobre las cláusulas abusivas en los contratos celebrados con consumidores, cuando el consumidor, ni en el propio procedimiento ejecutivo ni en un procedimiento judicial separado, puede obtener una tutela jurídica efectiva para ejercitar los derechos reconocidos en dicha Directiva, por ejemplo mediante una resolución judicial que suspenda provisionalmente la ejecución forzosa.» Este dictamen ha significado un ante y un después en los procedimientos hipotecarios, obligando a la administración de justicia y al propio Estado español, a una revisión y modificación en cuanto a este tipo de procedimiento.

Ante toda esta serie de circunstancias, se hace necesaria la intervención de los estados en una política de vivienda amplia y coordinada, en donde se favorezca y se facilite el acceso a aquellos sectores con mayor dificultad por cuestiones económicas, de accesibilidad, cultural, etc., para que la falta de una vivienda no se sitúe como factor que potencie un proceso de exclusión, sino por el contrario sea un instrumento preventivo y de refuerzo de 
cara a los ciudadanos en su proyecto de vida. «La vivienda es un espacio vital, imprescindible para la socialización, para ser parte de una sociedad, es decir, es un factor de inclusión social de primer orden». (Alguacil, et al., 2013, p. 4)

Al objeto de evitar que la vivienda sea utilizada como mera mercancía para la especulación, se propone partir de una regulación rígida de cara al mercado inmobiliario, respecto a las viviendas de primer uso para familias y/o personas a nivel individual, que inician un proceso de desarrollo personal, en donde la vivienda sea una de las herramientas que les facilita no sólo habitabilidad sino también seguridad, protección y espacio vital. La Declaración Universal de Derechos Humanos, como ya se ha indicado, establece el derecho a una vivienda digna, ya que este es uno de los componentes importantes para alcanzar un nivel de vida adecuado. (Mira, 2001).

Desde este planteamiento, se hace necesario acercarnos a la realidad de los desahucios, explorando las circunstancias previas y actuales que en ella se están produciendo, y su influencia en el proceso de desarrollo personal de las familias afectadas. Precisamente, esta es la finalidad de la presente investigación, con la que ha sido posible conocer y profundizar en las circunstancias que están originando el que las familias no puedan hacer frente al pago de su hipoteca, a la vez que, definir cuál es el perfil de éstas personas y/o familias.

\section{Material y Método}

\section{Participantes}

La muestra de participantes se ha diseñado a partir del informe sobre «Panorama registral Impagos hipotecarios de vivienda 2012», del Colegio de Registradores de la Propiedad, Bienes Muebles y Mercantiles de España, referenciado a la provincia de Málaga, en la que durante el año 2012 se han producido 2.268 adjudicaciones por ejecución hipotecaria. De todas estas adjudicaciones, el $77,06 \%$ corresponde a primera vivienda, lo que supone un total de 1.748. Sobre este dato se ha calculado el tamaño de la muestra para universos finitos con un nivel de confianza del $95 \%$ y un error del $\pm 9 \%$, resultando un total de 110 sujetos a quienes se les ha identificado de forma aleatoria, a partir de las personas que acuden a los diferentes recursos existentes en Málaga, para obtener información y/o asesoramiento sobre desahucios. 


\section{Instrumentos}

Se ha elaborado un cuestionario ${ }^{15}$ específico para la presente investigación, de 27 ítems, en los que a partir de preguntas tanto abiertas como cerradas se ha obtenido información sobre datos relativos a la composición y relaciones de la unidad familiar, datos económicos, sociodemográficos, de salud y afrontamiento del problema, respecto al cómo y dónde actuar para encontrar una salida, de relación con la entidad bancaria y situación de la vivienda con la hipoteca.

\section{Procedimiento}

El cuestionario diseñado se ha entregado en los diferentes organismos y entidades, en los que en estos momentos se está atendiendo a personas con problemas de desahucio en la ciudad de Málaga, en concreto se ha coordinado su distribución a través de la Oficina de Intermediación Hipotecaria de la Junta de Andalucía, trabajadores sociales de los Servicios Sociales Comunitarios de la provincia, Cáritas Diocesana, Cruz Roja y la Plataforma de Afectados por la Hipoteca (PAH). La recogida de datos se ha realizado de forma directa en la PAH de Málaga, participando en sus asambleas a lo largo de siete semanas. En el resto de entidades durante el mismo periodo, se ha contado con la colaboración de los técnicos y/o personas que atienden a los afectados para la aplicación del cuestionario. En todos los casos, se ha informado de la confidencialidad de los datos, así como del procedimiento voluntario para la participación en el estudio.

El porcentaje mayor de cuestionarios se ha obtenido a través de la $\mathrm{PAH}$, en concreto el $76,4 \%$, lo que puede estar explicado tanto por la recogida directa, como por ser dicha plataforma el mayor referente actual para la realidad de los desahucios en Málaga.

\section{Resultados}

Composición de la unidad familiar, datos económicos y sociodemográficos

Los resultados muestran que las familias que se encuentran actualmente en procesos de desahucios, están compuestas por dos adultos, con una media de edad de 40 años, y en un 59,1\% de los casos, con un menor de 9 años a su cargo.

El 89,1\% de dichas familias son de nacionalidad española.

15. Anexo 1 
Figura I. Familias en procesos de desahucios según nacionalidad
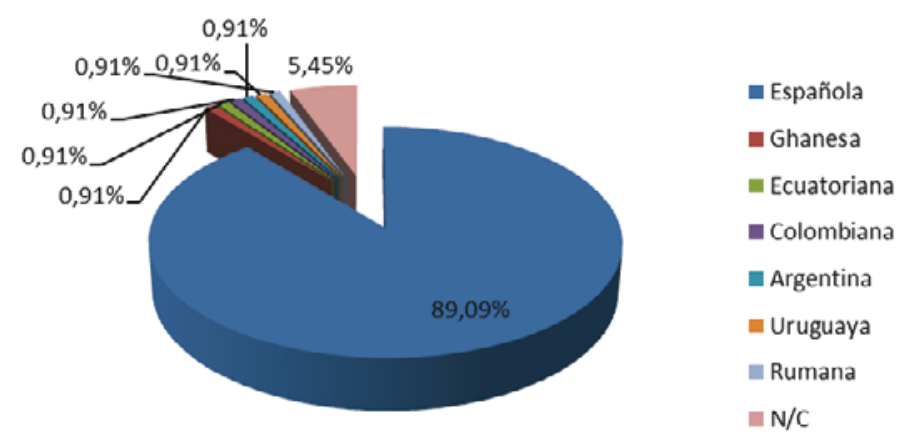

Para el 63,6\% de estas familias, la situación de desempleo es lo que está impidiendo hacer frente al pago de la hipoteca, seguidas por un 15,5\% de casos en los que tenían un negocio que se han visto obligados a cerrar.

Figura II. Circunstancia principal por la que no puede hacer frente a la cuota hipotecaria

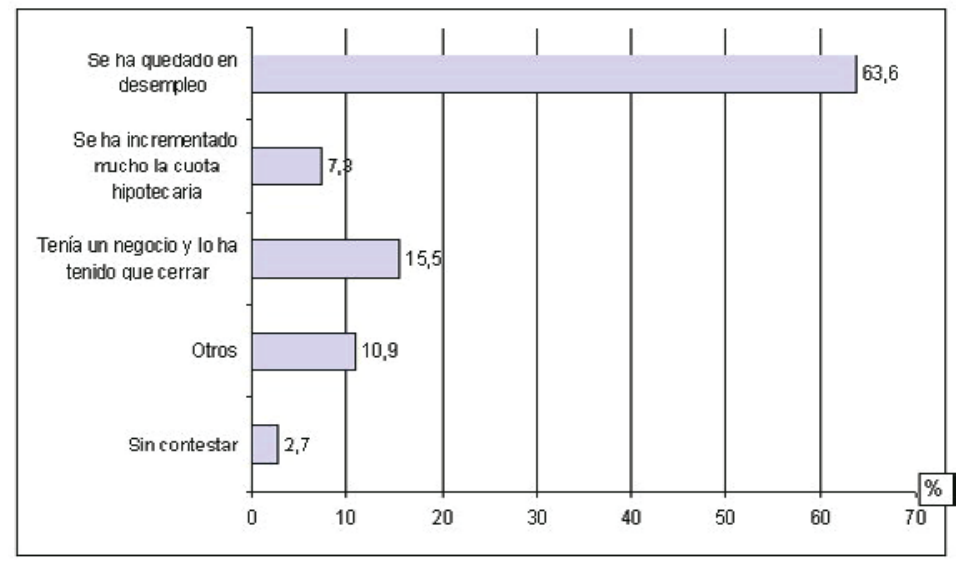

El 37,3\% de los participantes, en el momento de solicitar su hipoteca se encontraban trabajando en la construcción y en empresas auxiliares, en la hostelería y comercio un 32,4\%. La relación laboral que mantenían respondía en un $38,8 \%$ de los casos a un contrato temporal, seguido por un $36,9 \%$ en los que existía un contrato fijo y por un $19,4 \%$ que se encontraban de alta como autónomos. 
Figura III. Tipo de relación laboral que mantenía cuando le concedieron la hipoteca

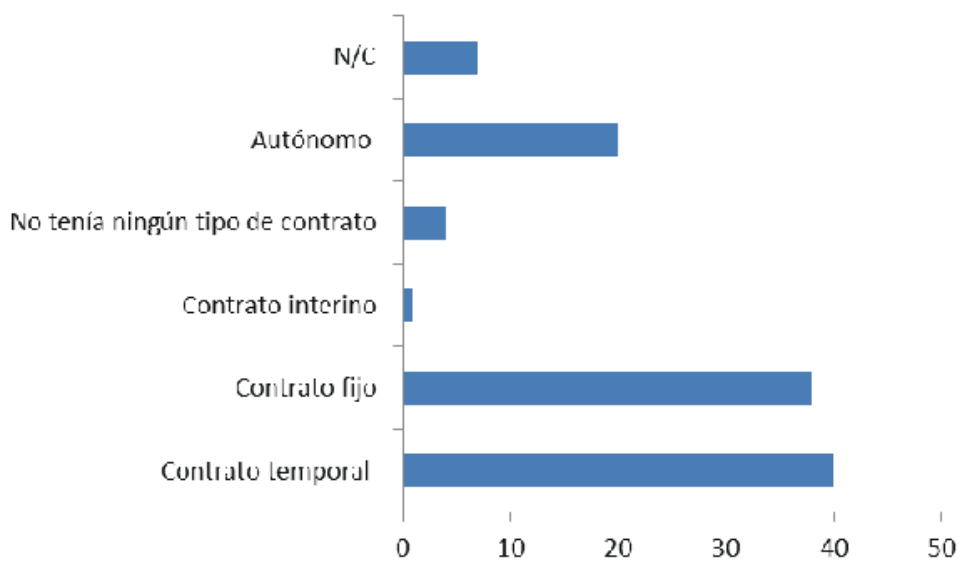

En el 35,1\% de las familias analizadas, los miembros que solicitaron la hipoteca llevaban más de diez años trabajando cuando se la concedieron, seguidos de entre 3 y 6 años en un 27,8\% de los casos, y con menos de un año en el $7,2 \%$ de ellos. En dichas familias se encontraban trabajando sólo un miembro de la unidad familiar, en ese momento, en el 49,5\% de los casos, y dos miembros en el $47,5 \%$.

Los ingresos mensuales que percibían cuando le concedieron la hipoteca se sitúan entre los 1.000 y 1.500 euros en un $37,5 \%$, y entre 1.500 y 2.000 euros en un $25 \%$. No superando los 1.000 euros de ingresos en el $16,3 \%$ de los casos.

Cuadro V. Ingresos económicos mensuales cuando le concedieron la hipoteca

\begin{tabular}{|l|r|}
\hline & $\%$ \\
\hline Entre 600 y 1.000 euros & 15.5 \\
\hline Entre 1.000 y 1.500 euros & 35.5 \\
\hline Entre 1.500 y 2000 euros & 23.6 \\
\hline Más de 2.000 euros & 20.0 \\
\hline N/C & 5.5 \\
\hline Total & 100.0 \\
\hline
\end{tabular}


En relación a la intención de adquirir una vivienda pública antes de acceder a una de renta libre vía hipoteca, el $72,1 \%$ de las personas encuestadas manifiestan no haberse planteado dicha opción, fundamentalmente por falta de información y por considerar que no reunían los requisitos exigidos para ello, dadas sus condiciones laborales, familiares y/o económicas. Respecto a si se plantearon el alquiler como una opción mejor que la compra, a través de la hipoteca, el 72,6\% de los participantes respondió que no, motivado principalmente por los precios del alquiler, excesivos y parecidos al de las hipotecas, y por el deseo de seguridad de cara al futuro.

Estado de salud, afrontamiento y actuación ante el problema

Los resultados muestran que, ante la imposibilidad de hacer frente al pago de la cuota hipotecaria, todos los participantes han experimentado sentimientos de miedo, ansiedad, culpa o la combinación entre ellos. Un 59,6\% de ellos, ha expresado que ha utilizado fármacos o consumido otras sustancias a raíz de no poder pagar la cuota hipotecaria. Y un $66,7 \%$ ha indicado que ha tenido problemas de salud, cuando tuvo conocimiento del desahucio, siendo principalmente éstos de ansiedad y depresión.

Figura IV. Problemas de salud presentados al tener conocimiento del desahucio

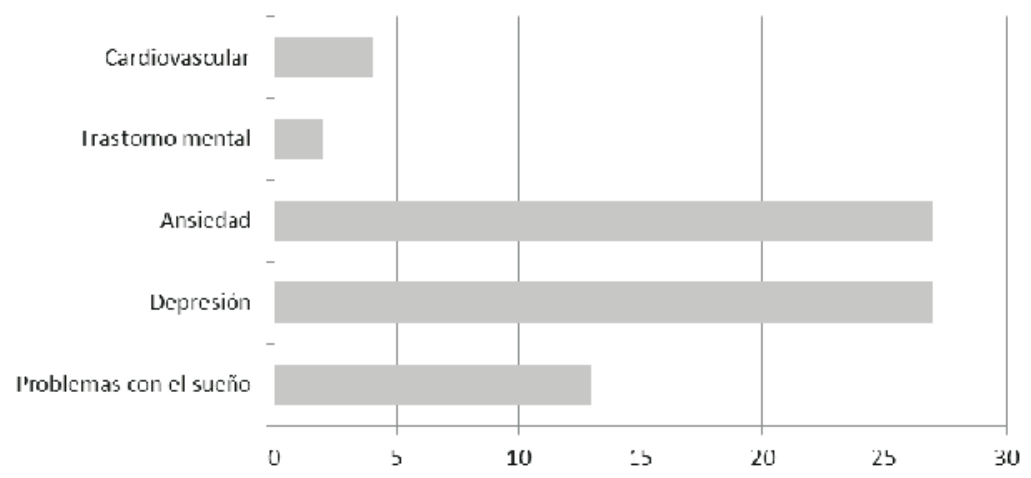

Así mismo, el 65,2\% de las familias reconocen haber experimentado cambios en su relación familiar tras la imposibilidad de hacer frente a la hipoteca. Éstos, en un 32,65\% de las situaciones, son definidos por ellos mismos como incremento de la agresividad, enfrentamientos y discusiones. 
Figura V. Cambios en la relación familiar ante la imposibilidad de hacer frente a la hipoteca

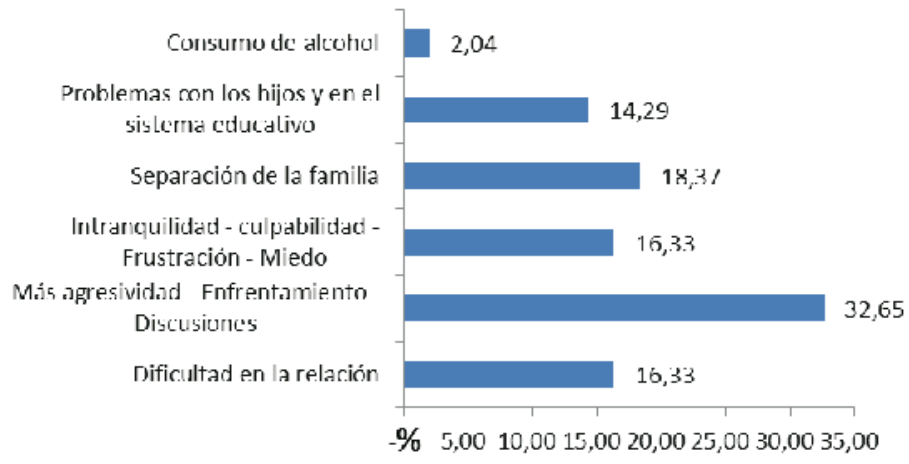

De cara a la actuación ante el problema, en un $43 \%$ de los casos, los participantes han acudido a los Servicios Sociales cuando han tenido dificultades para hacer frente a la cuota hipotecaria. También la familia, en un 36,4\% de las situaciones ha sido la primera opción en la que pensaron para solicitar su ayuda, encontrándose además un $28,3 \%$ de los casos, que no pensaron en nadie.

Hipoteca de la vivienda y relación con la entidad bancaria

El importe de la hipoteca solicitado se elevó a más de 200.000 euros en el $22,2 \%$ de los participantes, seguido de entre 100.000 y 125.000 euros en el $21,2 \%$. La media de años en que se encuentran financiadas estas hipotecas alcanza los 30,22 años. Estas hipotecas, en el 63,9\% de los casos no fueron avaladas por nadie, y entre quienes sí necesitaron aval, los madres/padres actuaron de avalista en el $56,4 \%$ de ellos y los hermanos en el $17,9 \%$.

En el 44,8\% de los casos, las entidades bancarias concedieron más importe en la hipoteca del solicitado previamente, manifestando el 68,3\% de los participantes haber dedicado dicho excedente a adquirir otros bienes de consumo o servicios, tales como reformar la vivienda o adquirir un automóvil. Tras la concesión de la hipoteca, en el 63,5\% de los casos, no se ha producido ampliación de la misma, ni a más años ni a más importe, mientras que en el 16,7\% de los mismos, sí que se ha producido el incremento a más importe, en el $14,6 \%$ a más años y en el 5,2\%, tanto a más años como a más importe.

El 39,8\% de estas familias, indican que la actitud de la entidad financiera cuando se ha producido una cuota devuelta, o cuando se le ha informado de que no se iba a poder pagar la cuota hipotecaria, ha sido autoritaria, junto a 
un $26,9 \%$ de los casos, en los que no han obtenido ninguna respuesta. Según los resultados, el $68,8 \%$ de las familias en procesos de desahucios, se han sentido acosadas por la entidad financiera o por otras empresas que actúan en su nombre. Situación ésta, que si la ponemos en relación con aquellas personas que han indicado que tuvieron necesidad de tomar fármacos o consumir algún tipo de sustancias, a raíz de no poder hacer frente a la hipoteca, obtenemos que son las que más han consumido, un $77,77 \%$, siendo por el contrario las que no se han sentido acosadas las que menos han consumido.

Figura VI. Relación entre personas que se han sentido acosada y las que expresan que han consumido algún fármaco o sustancia

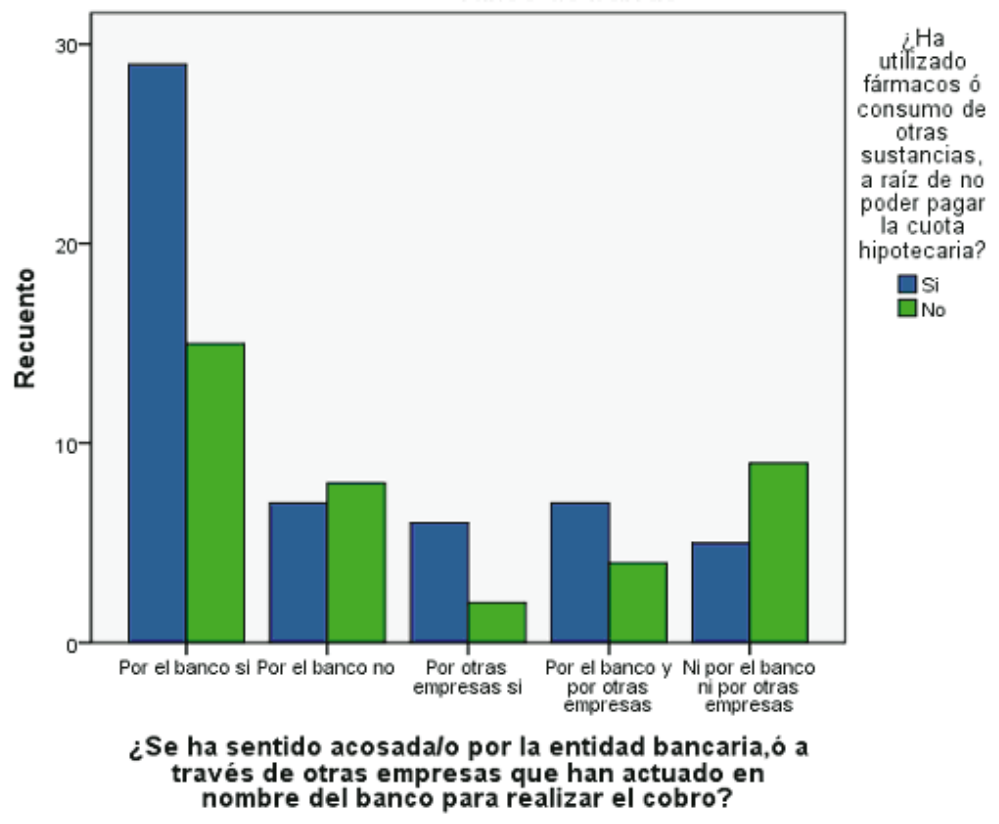

\section{Discusión}

El modelo de crecimiento planteado desde los años 50 en nuestro país, ha estado marcado por el peso específico de la construcción de vivienda privada por encima de la pública, así como por el cambio respecto al régimen de tenencia de la vivienda, pasando de la tendencia a la residencia en formato de alquiler a la vivienda en propiedad. Todo ello llega a su cenit con el llamado «boom inmobiliario». En este escenario, se ha ido originando un perfil de ciudadanos, cuya gran mayoría ha dirigido su actividad hacia la construcción 
o empresas auxiliares. A la vez, las entidades financieras han ido focalizando su negocio principal en todo lo relacionado con el sector inmobiliario, en muchos de los casos sin tener en cuenta medidas preventivas, y asumiendo un riesgo excesivo a la hora de conceder hipotecas a las familias españolas. De igual forma, las entidades locales se han sumado a este modelo, viendo en ello una fuente casi «inagotable» de ingresos financieros.

Con el inicio de la crisis financiera, como un castillo de naipes, este modelo de crecimiento ha caído en picado. La historia no sólo nos debiera de servir para conocer hechos y acontecimientos ocurridos, sino también para aprender de los aciertos y errores pasados, de cara a continuar o no en la realización de los mismos.

Por los datos obtenidos en este estudio, el hecho de que miles de ciudadanos se hayan quedado y se sigan quedando en desempleo y/o viéndose obligados a cerrar sus negocios, está provocando que en la gran mayoría de los casos analizados, éstos no puedan hacer frente al pago de la hipoteca.

Según los datos facilitados por el gobierno español, y remitidos a la comunidad europea a través de la «Actualización del Programa de Estabilidad 2013-2016» ${ }^{16}$, no se prevé que a corto plazo estas circunstancias vayan a ser modificadas. En este informe, se indica que la tasa de paro podrá alcanzar este año el $27,1 \%$, y podrá situarse para el año 2015 en un $25,8 \%$, superando la del año 2012 que fue del $25 \%$.

Tal como además ha reflejado esta investigación, y muy unido a lo anterior, nos encontramos ante un perfil de personas que no pueden hacer frente al pago de su hipoteca, por circunstancias de índole laboral. Ciudadanos que principalmente trabajaban en empresas de la construcción y auxiliares, y en los que primaba la contratación temporal cuando le concedieron la hipoteca. Estos datos y la pérdida del empleo, refleja las características de inseguridad que esta situación ha supuesto para las familias con hipotecas. De ahí que el cien por cien de ellos, expresaran que han sentido miedo, ansiedad o culpa cuando no han podido hacer frente a la cuota hipotecaria.

De igual forma, los resultados han reflejado el riesgo asumido por las entidades financieras, así como el esfuerzo económico que ha supuesto para las familias el que los ingresos previos a la hipoteca procedieran sólo de un miembro de la unidad familiar, con ingresos entre 1.500 y 2.000 euros, y el préstamo hipotecario medio haya oscilado entre los 150.000 y $200.000 \mathrm{eu}-$ ros. Esto fue suficiente para que las entidades financieras concedieran dichos

16. http://www.lamoncloa.gob.es/NR/rdonlyres/EFC55817-6964-4425-BB57B263B2351558/234921/ActualizacinProgramaEstabilidad201316ECONOMA.pdf 
préstamos, incluso sin avalista, en la gran mayoría de los casos. Ha quedado comprobado que estas familias asumieron un alto riesgo, agravado aun más por la ampliación, en muchos casos, del importe solicitado. El número medio de años contratado en las hipotecas se ha establecido en 30, en un mercado laboral cada vez más inestable, con la revisión actual del sistema de pensiones que apuesta por alternativas de alargar la edad de jubilación, y disminuir el importe a cobrar, y con una tasa nacional de paro juvenil del $57,22 \%$, según la EPA. Todo ello hará que se perpetúen situaciones de inviabilidad, tanto del acceso a la vivienda como del pago de la misma.

En relación al perfil de las familias con problemas en el pago de sus hipotecas, según los datos del presente estudio, nos encontramos con familias compuestas por 3 miembros, con una edad media de 40 años y con un menor de unos 9 años en la mayoría de los casos. Este perfil está en relación con los datos facilitados por las últimas encuestas del INE, que establecen la edad media de la maternidad en los 32 años, con un número de hijos por mujer de 1,3 , lo que refleja que las familias con problemas en el pago de la hipoteca ahora, son familias que iniciaban un proceso de desarrollo personal cuando optaron por la contratación de la misma. Además, no intentaron adquirir una vivienda pública por falta de información, y tampoco optaron por la opción del alquiler ya que los precios eran parecidos, y les generaba mayor seguridad de cara al futuro tener una vivienda de propiedad.

Las familias se tienen que enfrentar a situaciones nuevas para las que no están preparadas, como es el hecho de no poder hacer frente a un compromiso hipotecario contraído. Esta situación se vive como un acto reprobable socialmente, que conlleva a su vez a un enfrentamiento ante las entidades financieras. Generando en las familias sensación de impotencia, incapacidad y miedo, ocasionándoles no sólo problemas de salud sino también inestabilidad en las relaciones familiares. Es preocupante, que la principal circunstancia de conflicto familiar sea el incremento de la agresividad, los enfrentamientos y las discusiones en familias, como ya se ha indicado, en las que en la mayoría de los casos existen menores con no más de 9 años, con el riesgo que ello conlleva de cara a su desarrollo personal y educativo.

En toda esta realidad, según nos indica la investigación, sigue siendo la familia el mayor colchón social ante la que se acude en los momentos de crisis, bien sea a la más cercana padres y madres como a la de su entorno.

La falta de estudios previos de estas características, sitúa a este trabajo como un primer acercamiento a una realidad visibilizada a raíz de la crisis financiera, pero que ya existía antes para todas aquellas familias que eran desalojadas de sus viviendas. Estas situaciones venían siendo atendidas por los 
Servicios Sociales, los cuáles facilitaban en la mayoría de los casos una ayuda económica de emergencia. En estos momentos, tanto por el volumen como por las características de las personas que están siendo objeto de desahucio, las respuestas ofrecidas no pueden seguir siendo las mismas.

Esta investigación presenta limitaciones metodológicas que han de ser tenidas en cuenta en futuros estudios, en la dirección de obtener una mayor implicación y coordinación en el análisis de esta realidad, por parte del conjunto de las administraciones que atienden a personas con problemas para hacer frente a la cuota hipotecaria, lo que permitirá un incremento de la muestra, que mejore los índices de error.

\section{Conclusiones}

Los resultados aquí expuestos nos indican que urge intervenir en el problema del desempleo que sufren estas familias, ofreciendo no sólo alternativas a la vivienda, sino también recursos suficientes y válidos ante su situación de desempleo. De lo contrario, se estará generando una doble vulnerabilidad, la falta de una vivienda y la falta de recursos suficientes para satisfacer sus necesidades básicas y/o primarias.

De acuerdo con el informe del Defensor del Pueblo «Crisis económica y deudores hipotecarios: actuaciones y propuestas del Defensor del Pueblo», es necesario que las entidades financieras asuman una actitud negociadora, donde la mediación y el diálogo superen las circunstancias sobrevenidas por parte de las familias, y se puedan renegociar nuevas condiciones que mejoren las prácticas mantenidas de falta de información, agotando previo a las ejecuciones hipotecarias, la vía extrajudicial de la mediación. A la vez que están obligadas a la eliminación de las llamadas cláusulas abusivas.

Por otro lado, tanto ellas, como las administraciones públicas, debieran de establecer mecanismos novedosos y alternativos, para facilitar el acceso a la vivienda, más allá de alargar el número de años en que se concede el préstamo hipotecario, ya que es un riesgo ante la inseguridad del mercado laboral y sus perspectivas futuras.

La información que se facilite tanto por las administraciones públicas como por parte de las entidades financieras, debe ser correcta, suficiente y rigurosa, en cuanto a la adquisición de vivienda. Ya que no ha primado el interés general de los ciudadanos, y si por el contrario, el interés comercial y empresarial de cara a obtener beneficios a costa de un derecho, como es el disponer de una vivienda.

Es necesario hacer un seguimiento de las familias que se ven afectadas por un problema de desahucio, ofreciendo apoyo psicosocial no sólo a las 
personas afectadas sino también a su red familiar, abordando la intervención con los menores existentes. Siendo necesario por parte de los Servicios Sociales, que se establezcan líneas de intervención diferencias y actualizadas a una nueva realidad.

A la vez es fundamental contar con estadísticas periódicas y específicas, no sólo sobre los desahucios-lanzamientos que se producen, sino sobre el conjunto de las circunstancias que les rodea.

Y unido a todo ello, se evidencia la necesidad de que se realicen las modificaciones legislativas oportunas, para que se garantice el derecho a la vivienda como un derecho fundamental.

\section{Referencias}

Alguacil, A., Alguacil, J., Arasanz, J., Fernández, G., Paniagua, J.L., Olea, S. y RENES, V. (2013). La vivienda en España en el siglo XXI. Madrid: Fundación FOESSA. Cáritas.

Colau, A. y Alemany, A. (2012). Vidas hipotecadas. Barcelona: Cuadrilátero de libros.

- Empobrecimiento y desigualdad social. (2013).

- VIII Informe del Observatorio de la Realidad Social. (2013) Madrid: Cáritas Española.

Laparra, M., Obradors, A., Pérez, B., Pérez, M., Renes, V., Sarasa, S., Subirats, J. y TRujillo, M. (2007). Una propuesta de consenso sobre el concepto de exclusión. Implicaciones metodológicas. Revista Española del Tercer Sector, 5, $15-57$.

MiRA, Fco. J., (2001). Territorio, hábitat y vivienda frente a la exclusión social. Alternativas. Cuadernos de Trabajo Social, 9, 241-262.

Naciones Unidas. Consejo de Derechos Humanos. (2008) Promoción y protección de todos los derechos humanos, civiles, políticos, económicos, sociales y culturales incluidos el derecho al desarrollo. New York.

NAVARRO, C. (2005). Indicadores de Vivienda y exclusión. Documentación Social, 138, 173-190.

TRABADA, E. (2012). El problema de la vivienda en una sociedad que se dualiza. Documentación Social, 165, 165-188.

Ayuntamiento de Málaga. Presupuesto 2013. Estado Consolidado. Recuperado el 18 de mayo de $2013 \mathrm{http}: / /$ presupuestos2013.malaga.eu/export/sites/default/ economia/presupuestos2013/portal/menu/seccion_0001/documentos/estado_consolidado.pdf

Banco de España. Nota informativa sobre la presentación de una nueva estadística de procesos de ejecución hipotecaria sobre viviendas. Recuperado el 12 de 
mayo de 2013 http://www.bde.es/f/webbde/GAP/Secciones/SalaPrensa/NotasInformativas/Briefing_notes/es/notabe10-05-13.pdf

Boletín Oficial de la Junta de Andalucía. Decreto-ley 6/2013, de 9 de abril, de medidas para asegurar el cumplimiento de la Función Social de la Vivienda. Recuperado el 15 de mayo de 2013 http://www.juntadeandalucia.es/boja/2013/69/BOJA13-069-00026-6120-01_00025059.pdf

Boletín Oficial del Estado. Real Decreto-ley 27/2012, de 15 de noviembre, de medidas urgentes para reforzar la protección a los deudores hipotecarios. Recuperado el 15 de mayo de 2013 http://www.boe.es/boe/dias/2012/11/16/pdfs/ BOE-A-2012-14115.pdf

Boletín Oficial del Estado. Real Decreto-ley 6/2012, de 9 de marzo, de medidas urgentes de protección de deudores hipotecarios sin recursos. Recuperado el 15 de mayo de 2013 http://www.boe.es/boe/dias/2012/03/10/pdfs/BOEA-2012-3394.pdf

Boletín Oficial del Estado. Real Decreto 233/2013, de 5 de abril, por el que se regula el Plan Estatal de fomento del alquiler de viviendas, la rehabilitación edificatoria, y la regeneración y renovación urbana, 2013-2016. Recuperado el 12 de mayo de 2013 http://www.boe.es/boe/dias/2013/04/10/pdfs/BOEA-2013-3780.pdf

Boletín Oficial del Estado. Resolución de 23 de abril de 2013, de la Secretaría de

Estado de Servicios Sociales e Igualdad. http://www.boe.es/boe/dias/2013/05/16/ pdfs/BOE-A-2013-5163.pdf

Centro de Investigaciones Sociológicas (CIS). Barómetro de abril. Avance de resultados. Recuperado el 15 de mayo de 2013 http://datos.cis.es/pdf/ Es2984mar_A.pdf

Ciudades para un futuro más sostenible. Ayudas a 71 familias: Red Igloo Barcelona. Recuperado el 13 de octubre de 2013 http://habitat.aq.upm.es/bpes/ che00/bpes343.es.html

Colegio de Registradores de la Propiedad, Bienes Muebles y Mercantiles de España. Panorama Registral de Impagos Hipotecarios de Vivienda. Recuperado el 12 de mayo de 2013 http://www.registradores.org/get?DS=6\&file=/Estadisticas/Panorama_registral_de_impagos_hipotecarios_de_vivienda_en_2012. pdf

Consejo General del Poder Judicial. Informes periódicos. Efecto de la crisis en los órganos judiciales. Recuperado el 12 de mayo de 2013 http://www.poderjudicial.es/cgpj/es/Temas/Estadistica_Judicial/Informes_estadisticos/ Informes_periodicos

Consejo General del Poder Judicial. La Justicia dato a dato. Recuperado el 07 de julio de 2013 http://www.poderjudicial.es/cgpj/es/Temas/Estadistica Judicial/ Analisis estadistico/La Justicia dato a dato/ 
Defensor del Pueblo. Crisis económica y deudores hipotecarios: actuaciones y propuestas del Defensor del Pueblo. Recuperado el 26 de mayo de 2013 http://www.defensordelpueblo.es/es/Documentacion/Publicaciones/monografico/Documentacion/Crisis_Hipotecas_2012.pdf

Instituto Nacional de Estadística. Censo de población y vivienda 2011. Edificios y viviendas. Datos provisionales. Recuperado el 12 de mayo de 2013 http:// www.ine.es/prensa/np775.pdf

Instituto Nacional de Estadística. Construcción de vivienda según calificación. Recuperado el 12 de mayo de 2013 http://www.ine.es/jaxi/tabla.do?path=/t38/ bme2/t07/a081/10/\&file=1300007.px\&type=pcaxis $\& \mathrm{~L}=0$

Instituto Nacional de Estadística. Encuesta de condiciones de vida 2012. Resultados provisionales. Recuperado el 12 de mayo de 2013 http://www.ine.es/jaxi/ tabla.do?path=/t25/p453/provi/l0/\&file=01002.px\&type=pcaxis\&L=0

Instituto Nacional de Estadística. Encuesta de Población Activa. Recuperado el 12 de mayo de $2013 \mathrm{http} / /$ www.ine.es/jaxi/menu.do? $\mathrm{L}=0 \&$ type=pcaxis\&path= $\% 2 \mathrm{Ft} 22 \% 2 \mathrm{Fe} 308 \_m n u \& f i l e=i n e b a s e$

La Moncloa. Gobierno de España. Programa Nacional de Reformas y actualización del Programa de Estabilidad. Recuperado el 26 de mayo de 2013 http://www.lamoncloa.gob.es/NR/rdonlyres/EFC55817-6964-4425-BB57B263B2351558/234921/ActualizacinProgramaEstabilidad201316ECONOMA.pdf

Ministerio de Fomento. Precios de la vivienda. Recuperado el 22 de mayo de 2013 http://www.fomento.gob.es/BE2/?nivel=2\&orden=35000000

Naciones Unidas Derechos Humanos. El derecho a una vivienda adecuada. Recuperado el 22 de mayo de 2013 http://www.ohchr.org/Documents/Issues/ Housing/A-67-286_sp.pdf

Tribunal de Justicia Europeo. Conclusiones de la abogada general Sra. Juliane Kokott. Asunto C-445/11. Mohamed Aziz contra Catalunyacaixa. Recuperado el 10 de octubre de 2013 http://curia.europa.eu/juris/document/document.jsf? text $=\&$ docid $=129481 \&$ pageIndex $=0 \&$ doclang $=$ es $\&$ mode $=$ req $\&$ dir $=\&$ \&occ $=$ first $\&$ part $=1 \&$ cid $=172995$

\section{Anexo 1. Cuestionario}

El presente cuestionario forma parte de un trabajo de investigación que se está realizando en el Máster de Investigación e Intervención Social y Comunitaria de la Universidad de Málaga.

La información aquí facilitada es total y absolutamente anónima. Su contestación no le llevará más de 15 minutos, sólo tendrá que marcar con una X la respuesta que considere oportuna. Agradeciendo su interés en participar contestando las siguientes preguntas. 
Número de personas que componen la Unidad Familiar

Edad de las personas de la Unidad Familiar
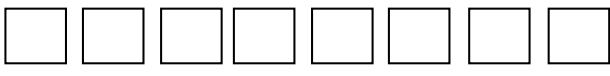

Nacionalidad

1) Ante la imposibilidad de hacer frente al pago de la cuota hipotecaria, ¿experimentó sentimientos de?

\begin{tabular}{|l|l|l|l|l|l|l|l|}
\hline Miedo & & Ansiedad & & Indiferencia & & Culpa & \\
\hline
\end{tabular}

Otros, indicar cuales

2) ¿Ha utilizado fármacos ó consumo de otras sustancias, a raíz de no poder pagar la cuota hipotecaria?

En caso afirmativo ¿cuáles?

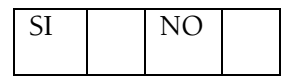

\begin{tabular}{|c|c|c|c|}
\hline Antidepresivos & Tranquilizantes & Alcohol & Consumo excesivo de café \\
\hline
\end{tabular}

Otros, indicar cuales

3) ¿Le avalo algún familiar y/o amigo para la concesión de la hipoteca?

En caso afirmativo ¿quién?

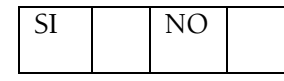

\begin{tabular}{|c|c|c|c|}
\hline Madres/padres & Hermana/o & Suegra/o & Amiga/o \\
\hline
\end{tabular}

Otros, indicar cuales

4) ¿La circunstancia principal por la que no puede hacer frente a la cuota hipotecaria es?

\begin{tabular}{|c|c|c|c|c|}
\hline $\begin{array}{c}\text { Se ha quedado en } \\
\text { desempleo }\end{array}$ & $\begin{array}{c}\text { Se ha incrementado } \\
\text { mucho la cuota }\end{array}$ & $\begin{array}{c}\text { Tenía un negocio y lo } \\
\text { ha tenido que cerrar }\end{array}$ \\
\hline
\end{tabular}

Otros, indicar cual 
5) ¿En que trabajaba cuando solicitó la hipoteca?

6) ¿Qué tipo de relación laboral mantenía cuando le concedieron la hipoteca?

\begin{tabular}{|c|c|c|c|c|c|c|c|c|}
\hline $\begin{array}{c}\text { Contrato } \\
\text { temporal }\end{array}$ & Contrato fijo & $\begin{array}{c}\text { Contrato } \\
\text { interino }\end{array}$ & $\begin{array}{c}\text { No tenía ningún } \\
\text { tipo de contrato }\end{array}$ & $\begin{array}{c}\text { Era } \\
\text { autónomo }\end{array}$ & \\
\hline
\end{tabular}

7) ¿Cuántos años llevaba trabajando cuando le concedieron la hipoteca?

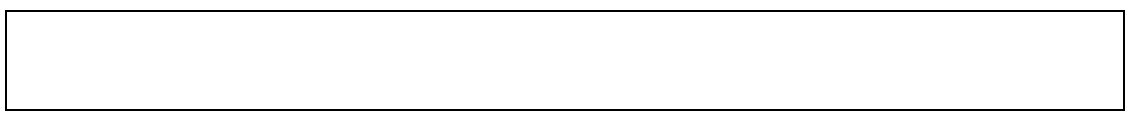

8) ¿Cuántos miembros de la unidad familiar trabajaban cuando le concedieron la hipoteca?

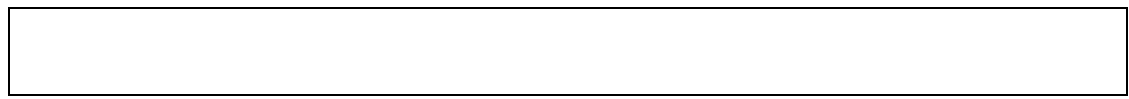

9) ¿Cuáles eran sus ingresos económicos mensuales cuando le concedieron la hipoteca?

\begin{tabular}{|c|c|c|c|c|c|c|}
\hline $\begin{array}{c}\text { Entre } 600 \mathrm{y} 1.000 \\
\text { euros }\end{array}$ & $\begin{array}{c}\text { Entre } 1.000 \mathrm{y} \\
1.500 \text { euros }\end{array}$ & $\begin{array}{c}\text { Entre } 1.500 \mathrm{y} \\
2.000 \text { euros }\end{array}$ & $\begin{array}{c}\text { Más de } 2.000 \\
\text { euros }\end{array}$ & \\
\hline
\end{tabular}

10) ¿Intentó adquirir una vivienda pública antes de adquirir una de renta libre vía hipoteca?

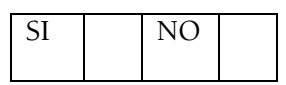

En caso de que la respuesta sea negativa, puede indicar el motivo

En caso de que la respuesta sea afirmativa, ¿por qué optó al final por la de renta libre?

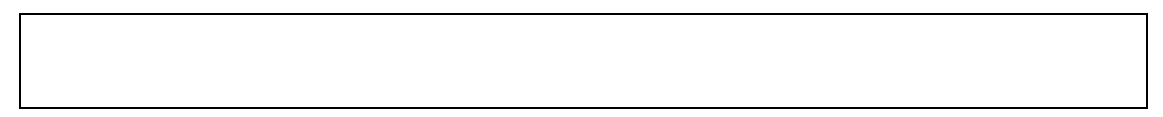


11) ¿Se planteó el alquiler como una opción mejor que la compra a través de una hipoteca?

\begin{tabular}{|l|l|l|l|}
\hline SI & & NO & \\
\hline
\end{tabular}

En caso de que la respuesta sea afirmativa, ¿por qué no lo hizo?

12) ¿Una vez que tuvo conocimiento del desahucio ha tenido problemas de salud provocados por ésta situación?

\begin{tabular}{|l|l|l|l|}
\hline SI & & NO & \\
\hline
\end{tabular}

En caso de que la respuesta sea afirmativa, puede indicar cuáles

13) ¿Se ha producido algún cambio en la relación familiar, de los miembros que conviven en el domicilio, tras la imposibilidad de hacer frente a la hipoteca?

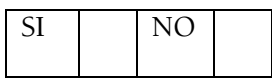

En caso de que la respuesta sea afirmativa, ¿puede indicar que cambios en la relación familiar son los que han ocurrido?

14) El comportamiento de la entidad bancaria, ante una cuota devuelta o cuando usted le ha informado que no iba a poder pagarla, ¿ha sido de?

\begin{tabular}{|l|l|c|c|c|c|c|c|c|}
\hline Comprensión & $\begin{array}{c}\text { Facilitadora } \\
\text { de soluciones }\end{array}$ & Negociadora & $\begin{array}{c}\text { No ha tenido } \\
\text { respuesta }\end{array}$ & Autoritaria & \\
\hline
\end{tabular}

Otro, indicar cual 
15) ¿Se ha sentido acosada/o por la entidad bancaria, ó a través de otras empresas que han actuado en nombre del banco para realizar el cobro?

\begin{tabular}{|l|l|l|l|l|l|l|l|l|l|}
\hline Por el banco & SI & & NO & & Por otras empresas & SI & & NO & \\
\hline
\end{tabular}

16) ¿Cuándo solicitó el préstamo hipotecario le dieron el importe económico mínimo necesario para comprar la vivienda o más, del que usted necesitaba?

\begin{tabular}{|l|l|l|l|}
\hline Me dieron el Importe mínimo & & Me dieron más de lo que necesitaba & \\
\hline
\end{tabular}

17) ¿Adquirió algún otro bien de consumo: coche, TV... o servicio: hizo un viaje, una obra... al adquirir el préstamo hipotecario que se incorporó a la hipoteca?

\begin{tabular}{|l|l|l|l|}
\hline SI & & NO & \\
\hline
\end{tabular}

En caso de ser afirmativo, podría indicar ¿cuál?

18) ¿Recuerda cuántos años firmó de hipoteca?

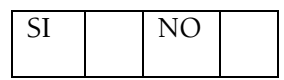

En caso afirmativo puede indicar ¿cuántos?

19) ¿Qué cantidad solicitó de hipoteca?

\begin{tabular}{|c|c|c|c|c|c|c|c|c|}
\hline $\begin{array}{c}\text { Menos de } \\
100.000 \\
\text { euros }\end{array}$ & $\begin{array}{c}\text { Entre } \\
100.000 \mathrm{y} \\
125.000 \\
\text { euros }\end{array}$ & $\begin{array}{c}\text { Entre } \\
125.000 \mathrm{y} \\
150.000 \\
\text { euros }\end{array}$ & $\begin{array}{c}\text { Entre } \\
150.000 \mathrm{y} \\
175.000 \\
\text { euros }\end{array}$ & $\begin{array}{c}\text { Entre } \\
175.000 \mathrm{y} \\
200.000 \\
\text { euros }\end{array}$ & $\begin{array}{c}\text { Más de } \\
200.00 \\
\text { euros }\end{array}$ \\
& & & & & \\
\hline
\end{tabular}

20) ¿Ha realizado alguna ampliación de la hipoteca en más años o a más importe?

\begin{tabular}{|l|l|l|l|l|}
\hline Si a más años & Si a más importe & & No ni a más años ni a más importe & \\
\hline
\end{tabular}

21) ¿Ha acudido a los servicios sociales de su Ayuntamiento, cuando no ha podido hacer frente a la cuota hipotecaria?

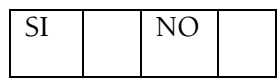


En caso de que la respuesta sea negativa, puede indicar ¿por qué?

En caso afirmativo ¿qué respuesta le han dado?

22) ¿Quién pensó primeramente que le podía ayudar cuando no pudo hacer frente al pago de la cuota hipotecaria?

23) ¿Se encuentra inscrita/o en el Registro de demandante de vivienda de su ayuntamiento?

\begin{tabular}{|l|l|l|l|}
\hline SI & & NO & \\
\hline
\end{tabular}

En caso de que la respuesta sea negativa puede indicar ¿por qué?

24) ¿Cuál cree que podría ser una solución para no tener que ser desahuciada/o?

MUCHAS GRACIAS POR SU TIEMPO Y SUS RESPUESTAS

Alternativas, 20, 2013, pp. 113-140 - ISSN 1133-0473 - DOI: 10.14198/ALTERN2013.20.07 